\title{
Effect of Age of Onset of Psoriasis on Clinical Outcomes with Systemic Treatment in the Psoriasis Longitudinal Assessment and Registry (PSOLAR)
}

\author{
Sanminder Singh ${ }^{1} \cdot$ Robert E. Kalb ${ }^{2}$ - Elke M. G. J. de Jong ${ }^{3} \cdot$ Neil H. Shear $^{4} \cdot$ Mark Lebwohl $^{5} \cdot$ Wayne Langholff $^{6}$. \\ Lori Hopkins $^{7} \cdot$ Bhaskar Srivastava $^{7} \cdot$ April W. Armstrong $^{8}$
}

Published online: 28 September 2018

(c) The Author(s) 2018

\begin{abstract}
Objective Our objective was to compare therapeutic response among patients with early-onset psoriasis (EOP) and late-onset psoriasis (LOP) receiving adalimumab, etanercept, infliximab, ustekinumab, or methotrexate in the Psoriasis Longitudinal Assessment and Registry (PSOLAR).

Methods Patients were grouped by age of onset: EOP (age $\leq 40$ years) or LOP (age $>40$ years). Repeated-measures analysis with logistic regression was used to calculate the adjusted odds ratio (AOR; adjusted for baseline characteristics) for achieving a Physician's Global Assessment score of cleared/minimal (PGA 0/1) or a percentage of body surface area involved with psoriasis $<3 \%(\% \mathrm{BSA}<3)$ or \% BSA $<1$ for all patients; similar sensitivity analyses were performed for each treatment group. Results Of 7511 patients, 5479 (72.9\%) had EOP. The LOP group had a higher likelihood of achieving PGA 0/1 after treatment than did the EOP group in all patients (AOR 1.14 [95\% confidence interval (CI) 1.05-1.25]; $p=0.0019$ ); the same was true in subgroups of etanercept-treated (AOR 1.38 [95\% CI 1.14-1.66]; $p=0.0010$ ) and methotrexate-treated (AOR 1.62 [95\% CI 1.16-2.26]; $p=0.0049$ ) patients. No significant difference was found between the EOP and LOP groups with regard to the likelihood of achieving $\% \mathrm{BSA}<3$ or $\% \mathrm{BSA}<1$ among all patients. However, LOP patients were more likely than EOP patients to achieve \%BSA $<3$ or \%BSA $<1$ in subgroups treated with infliximab (AOR 1.45 [95\% CI 1.09-1.93; $p=0.0103$ ] and AOR 1.36 [95\% CI 1.03-1.78; $p=0.0290$ ], respectively) and etanercept (AOR 1.30 [95\% CI 1.06-1.61; $p=0.0123]$ and AOR 1.34 [95\% CI 1.09-1.64; $p=0.0053]$, respectively).

Conclusion Our real-world data from PSOLAR indicate that there are differences in some patient characteristics between EOP and LOP and that patients with EOP are less likely than those with LOP to respond to certain systemic treatments. (ClinicalTrials.gov identifier: NCT00508547).
\end{abstract}

April W. Armstrong

aprilarmstrong@post.harvard.edu

1 Davis School of Medicine, University of California, Sacramento, CA, USA

2 State University of New York at Buffalo School of Medicine and Biomedical Sciences, Buffalo, NY, USA

3 Radboud University Medical Center and Radboud University, Nijmegen, The Netherlands

4 Sunnybrook Health Sciences Center, University of Toronto Medical School, Toronto, ON, Canada
5 Icahn School of Medicine at Mount Sinai, New York, NY, USA

6 Janssen Research \& Development, LLC, Horsham, PA, USA

7 Janssen Scientific Affairs, LLC, Horsham, PA, USA

8 Keck School of Medicine, University of Southern California, 1975 Zonal Avenue, Keith Administration Building, Room 510, Los Angeles, CA 90089, USA 


\section{Key Points}

Based on a review of over 7500 patients enrolled in the PSOLAR registry, some disease characteristics may differ between early-onset psoriasis and late-onset psoriasis (aged $\leq 40$ years and $>40$ years, respectively).

Results of modeled analyses adjusted for differences in baseline characteristics showed that response to certain biologic agents, such as infliximab and etanercept, is better in late-onset psoriasis than in early-onset psoriasis.

Age of onset may be an important consideration in developing individualized treatment regimens to maximize therapeutic response for patients with psoriasis.

\section{Introduction}

Psoriasis is a chronic, inflammatory skin condition that affects between 2 and $4 \%$ of the global population and varies widely between countries [1]. Psoriasis onset may occur at any age; however, some studies have demonstrated a bimodal distribution of age of onset in both male and female patients with psoriasis. One peak occurred at age 16 years for females and 22 years for males, with another peak at age 60 years for females and 57 years for males [2]. Two subtypes of psoriasis have been identified based on age of onset: early-onset psoriasis (EOP; age $\leq 40$ years) and late-onset psoriasis (LOP; age $>40$ years) [2]. The dichotomizing of psoriasis patients into early versus late onset of disease is consistent with differences in genetic predisposition and clinical presentation.

Understanding the differences between these two subtypes of psoriasis is important because they appear to manifest with variable disease activity and may be associated with different patterns of disease. The landmark study by Henseler et al. [2] found that patients with EOP were more likely than those with LOP to experience frequent disease relapses (73 vs. 27\%), extensive body surface involvement ( 75 vs. $25 \%$ ), and nail involvement (74 vs. $26 \%$ ). The authors also reported a higher prevalence of EOP versus LOP when stratified by sex (i.e., 74 vs. $26 \%$ for males and 66 vs. $34 \%$ for females) [2]. EOP and LOP are further characterized by differences in genetic susceptibility loci. For instance, EOP has been strongly associated with human leukocyte antigen (HLA)-Cw6, HLA-B57, and HLA-DR7 alleles, whereas the HLA-Cw2 allele is overrepresented in LOP [2, 3]. Additionally, patients with EOP are more likely to have a positive family history and require systemic therapies than are those with LOP [4].
As the number of options for treating psoriasis continues to grow, there is increasing focus on how clinicians can provide patient-directed therapies to maximize treatment outcomes. One study found differences in response to etanercept between patients with EOP and those with LOP, but the study was limited by the number of treatments and sample size [5]. Real-world evidence of treatment outcomes in patients with EOP and LOP is lacking in the literature. The Psoriasis Longitudinal Assessment and Registry (PSOLAR) is a prospective, longitudinal, noninterventional, disease-based registry designed to collect clinical and safety outcome data from patients receiving systemic or biologic therapies through and up to 8 years $[6,7]$. In this study, we used PSOLAR data to examine differences in treatment response between EOP and LOP following treatment with a biologic agent (i.e., adalimumab, etanercept, infliximab, ustekinumab) or methotrexate.

\section{Methods}

\subsection{Study Design and Patients}

The design of PSOLAR has been reported previously [6, 7]. Briefly, patients had to be aged $\geq 18$ years and must have had a diagnosis of psoriasis, for which they were receiving, or were eligible to receive, treatment with systemic therapies as prescribed by their physician per actual clinical practice. Data (e.g., demographic and patient characteristics, clinical and safety outcomes, and psoriasis treatment) are collected every 6 months. Enrollment in PSOLAR began on 20 June 2007 , and the registry is fully enrolled (12,090 patients in 16 countries); the data included in this analysis were collected through 23 August 2015. Planned follow-up for each patient is 8 years from the time of registry enrollment.

\subsection{Ethics}

The registry (ClinicalTrials.gov identifier: NCT00508547) is conducted in accordance with current US FDA regulations and guidelines, International Conference on Harmonization Good Clinical Practices, the principles of the Declaration of Helsinki, and all other applicable national and local laws and regulations. The study protocol was approved by an institutional review board or ethics committee at all sites, and written informed consent was provided by all patients before study procedures were initiated.

\subsection{Outcome Measures}

Treatment outcomes were measured based on two standard assessments of psoriasis severity: Physician's Global Assessment (PGA) scores and percentage of body surface 
area involved with psoriasis (\%BSA). The PGA evaluates the qualitative characteristics of psoriasis lesions (i.e., induration, scaling, and erythema), resulting in a total score ranging from clear (0) to severe (5).

\subsection{Statistical Analyses}

To be included in the analysis, patients had to have received systemic treatment with a biologic agent (adalimumab, etanercept, infliximab, or ustekinumab) or methotrexate within 6 months of enrollment in the registry. In addition, data regarding the age of psoriasis onset as well as baseline (pretreatment) PGA scores or \%BSA must have been recorded within the 3 months before starting therapy. Patients were grouped by their age of onset: EOP (age $\leq 40$ years) or LOP (age $>40$ years). The proportions of patients achieving a PGA score of 0 or $1(\mathrm{PGA} 0 / 1)$ or a $\% \mathrm{BSA}<3$ or $\% \mathrm{BSA}<1$ were summarized for the EOP and LOP groups at four post-baseline visits (i.e., 6 months, 1 year, 1.5 years, and 2 years) for the overall population and for each treatment group (etanercept, adalimumab, infliximab, ustekinumab, or methotrexate). Repeated-measures analysis with logistic regression was used to calculate the adjusted odds ratio (AOR) for achieving PGA $0 / 1$ and $\% \mathrm{BSA}<3$ or $\% \mathrm{BSA}<1$ for the overall population. Similar sensitivity analyses were performed for each treatment group. Beyond the age-ofonset group (EOP or LOP) variables, potential confounders in the modeled analyses included baseline PGA $(0-1,2-3$, $4-5)$ or \%BSA $(0-3,>3-<10, \geq 10)$, current treatment, postbaseline visit ( 6 months, 1 year, 1.5 years, 2 years), baseline body mass index, race, self-reported psoriatic arthritis, past or current smoking history, and past treatment with biologics (ustekinumab, adalimumab, etanercept, or infliximab), oral systemic agents (including methotrexate), and phototherapy.

\section{Results}

\subsection{Patient Disposition and Characteristics}

Of the 12,090 patients enrolled in the registry, 7511 were eligible for inclusion in this analysis. The median duration of follow-up as of the data cut-off date for this analysis was 4.17 years (maximum 8.12 years). Patients with EOP accounted for $72.9 \%$ (5479/7511) of the total sample size (Table 1). Most patients in the study population were enrolled at sites located in North America (88.7\%) (Table 1). Mean age was 44.8 years in the EOP group and 60.2 years in the LOP group. More than half of the patients in each group were male $(57.7 \%$ [EOP] and $52.4 \%$ [LOP]), and most patients were White (84.3\% [EOP] and $80.8 \%$ [LOP]). Most patients had a diagnosis of plaque psoriasis (98.0 and 95.9\% for EOP and LOP, respectively); other diagnoses, which were not mutually exclusive, included guttate, erythrodermic, pustular, and/or inverse psoriasis. Baseline disease severity for the overall population was moderate in most patients, as measured by PGA (53.3\% had a score of 2-3) and $\%$ BSA (mean \pm standard deviation, $11.5 \pm 17.1$ ); more than one-third of patients (38.5\%) had a self-reported history of psoriatic arthritis.

In general, disease characteristics were consistent across the EOP and LOP groups, with a few exceptions. As expected, mean disease duration was higher in the EOP than in the LOP groups (21.8 vs. 8.3 years). A higher proportion of EOP than LOP patients reported a family history of psoriasis ( 47.5 vs. $36.9 \%$ ) and a history of use of oral systemic treatment (34.8 vs. $29.3 \%$ ), phototherapy (61.6 vs. $40.2 \%$ ), and biologic agents ( 82.8 vs. $73.6 \%$ ) for psoriasis prior to registry enrollment.

Overall, $37.6 \%$ of patients were exposed to ustekinumab, $11.7 \%$ to infliximab, $18.2 \%$ to etanercept, $27.0 \%$ to adalimumab, and $5.5 \%$ to methotrexate. Compared with the EOP group, fewer patients with LOP were exposed to ustekinumab (40.2 vs. $30.6 \%$ ) and more patients with LOP were exposed to methotrexate (3.6 vs. $10.5 \%$ ).

\subsection{Clinical Outcomes}

\subsubsection{Physician's Global Assessment}

There were no differences in the proportions of patients achieving a PGA 0/1 response at 6 months, 1 year, 1.5 years, and 2 years between the EOP and LOP groups (Table 2). After adjusting for potential confounders, patients in the LOP group were more likely to have achieved a PGA 0/1 response than those in the EOP group (AOR 1.14 [95\% confidence interval (CI) 1.05-1.25]; $p=0.0019)$. Consistent results were observed in the sensitivity analysis of patients treated with etanercept (AOR 1.38 [95\% CI 1.14-1.66]; $p=0.0010$ ) or methotrexate (AOR 1.62 [95\% CI 1.16-2.26]; $p=0.0049$ ). Modeled findings for adalimumab, infliximab, or ustekinumab showed no differences in the likelihood of patients achieving PGA 0/1 between the EOP and LOP groups (Table 2).

\subsubsection{Percent Body Surface Area}

The proportions of patients achieving $\% \mathrm{BSA}<3$ or $\% \mathrm{BSA}<1$ were similar for the EOP and LOP groups at 6 months, 1 year, 1.5 years, and 2 years (Tables 3 and 4). Based on AORs, no significant difference in the likelihood of achieving $\% \mathrm{BSA}<3$ or $\% \mathrm{BSA}<1$ was found between the EOP and LOP groups. However, sensitivity analyses showed that patients in the LOP group were more likely than those in the EOP group to achieve $\% \mathrm{BSA}<3$ and $\% \mathrm{BSA}<1$ when treated with infliximab or etanercept. The likelihood of LOP patients treated with infliximab achieving $\% \mathrm{BSA}<3$ was $45 \%$ greater (AOR 1.45 [95\% 
Table 1 Demographic and patient characteristics at enrollment by age of onset

\begin{tabular}{|c|c|c|c|}
\hline & $\operatorname{EOP}^{\mathrm{a}}(n=5479)$ & $\mathrm{LOP}^{\mathrm{b}}(n=2032)$ & All patients $(n=7511)$ \\
\hline \multicolumn{4}{|l|}{ Treatment $^{\mathrm{c}}$} \\
\hline Adalimumab & $1479(27.0)$ & $549(27.0)$ & $2028(27.0)$ \\
\hline Etanercept & $952(17.4)$ & $415(20.4)$ & $1367(18.2)$ \\
\hline Infliximab & $648(11.8)$ & $232(11.4)$ & $880(11.7)$ \\
\hline Ustekinumab & $2202(40.2)$ & $622(30.6)$ & $2824(37.6)$ \\
\hline Methotrexate & $198(3.6)$ & $214(10.5)$ & $412(5.5)$ \\
\hline Age, years & $44.8 \pm 12.3$ & $60.2 \pm 9.0$ & $49.0 \pm 13.4$ \\
\hline \multicolumn{4}{|l|}{ Age (years) at enrollment } \\
\hline Quartile 1 (18 to < 39) & $1877(34.3)$ & - & $1877(25.0)$ \\
\hline Quartile $2(\geq 39$ to $<50)$ & $1647(30.1)$ & $231(11.4)$ & $1878(25.0)$ \\
\hline Quartile $3(\geq 50$ to $<59)$ & $1176(21.5)$ & $702(34.6)$ & $1878(25.0)$ \\
\hline Quartile $4(\geq 59)$ & $779(14.2)$ & $1099(54.1)$ & $1878(25.0)$ \\
\hline Sex, male & $3160(57.7)$ & $1064(52.4)$ & $4224(56.2)$ \\
\hline \multicolumn{4}{|l|}{ Race } \\
\hline White & $4618(84.3)$ & $1641(80.8)$ & $6259(83.3)$ \\
\hline Black or African American & $151(2.8)$ & $105(5.2)$ & $256(3.4)$ \\
\hline Asian & $220(4.0)$ & $80(3.9)$ & $300(4.0)$ \\
\hline Hispanic or Latino & $351(6.4)$ & $154(7.6)$ & $505(6.7)$ \\
\hline Other & $139(2.5)$ & $52(2.6)$ & $191(2.5)$ \\
\hline \multicolumn{4}{|l|}{ Region } \\
\hline North America & $4817(87.9)$ & $1845(90.8)$ & $6662(88.7)$ \\
\hline Europe & $601(11.0)$ & $154(7.6)$ & $755(10.1)$ \\
\hline South America & $61(1.1)$ & $33(1.6)$ & $94(1.3)$ \\
\hline Family history of psoriasis & $2602(47.5)$ & 749 (36.9) & $3351(44.6)$ \\
\hline Plaque & $5367(98.0)$ & 1949 (95.9) & $7316(97.4)$ \\
\hline Other $^{\mathrm{d}}$ & $438(8.0)$ & $210(10.3)$ & $648(8.6)$ \\
\hline Guttate & $235(4.3)$ & $58(2.9)$ & $293(3.9)$ \\
\hline Erythrodermic & $51(0.9)$ & $31(1.5)$ & $82(1.1)$ \\
\hline Pustular & $55(1.0)$ & $76(3.7)$ & $131(1.7)$ \\
\hline Inverse & $127(2.3)$ & $60(3.0)$ & $187(2.5)$ \\
\hline Psoriatic arthritis, self-reported & $2105(38.4)$ & $789(38.8)$ & $2894(38.5)$ \\
\hline \multicolumn{4}{|l|}{ PGA score } \\
\hline $0-1$ & $2025(37.0)$ & $746(36.7)$ & $2771(36.9)$ \\
\hline $2-3$ & $2923(53.4)$ & $1083(53.3)$ & $4006(53.3)$ \\
\hline $4-5$ & $531(9.7)$ & $203(10.0)$ & $734(9.8)$ \\
\hline Mean BSA, \% (median) & $11.5 \pm 17.1(5.0)$ & $11.5 \pm 17.2(5.0)$ & $11.5 \pm 17.1(5.0)$ \\
\hline Mean duration of psoriasis, years (median) & $21.8 \pm 13.0(20.5)$ & $8.3 \pm 7.2(6.3)$ & $18.1 \pm 13.1(16.3)$ \\
\hline \multicolumn{4}{|l|}{ Prior treatment } \\
\hline Oral systemic agents & $1909(34.8)$ & $596(29.3)$ & $2505(33.4)$ \\
\hline Phototherapy & 3374 (61.6) & $816(40.2)$ & $4190(55.8)$ \\
\hline Biologics & $4539(82.8)$ & $1496(73.6)$ & $6035(80.3)$ \\
\hline
\end{tabular}

Data are presented as mean \pm standard deviation or number of patients (\%) unless otherwise indicated

$B S A$ body surface area, EOP early-onset psoriasis, $L O P$ late-onset psoriasis, $P G A$ Physician Global Assessment

${ }^{a}$ Age of onset $\leq 40$ years

${ }^{\mathrm{b}}$ Age of onset $>40$ years

${ }^{\mathrm{c}}$ First therapy on registry

${ }^{\mathrm{d}}$ Other types of psoriasis were not mutually exclusive 
Table 2 Patients achieving PGA 0/1 at post-baseline visits based on age of onset

\begin{tabular}{|c|c|c|c|c|c|c|c|c|c|}
\hline \multirow[t]{3}{*}{ Treatment } & \multicolumn{8}{|c|}{ Proportion of patients achieving PGA $0 / 1$} & \multirow{3}{*}{$\begin{array}{l}\text { Odds of achieving PGA 0/1 } \\
\text { for LOP vs. EOP }\end{array}$} \\
\hline & \multicolumn{2}{|c|}{ At 6 months } & \multicolumn{2}{|c|}{ At 1 year } & \multicolumn{2}{|c|}{ At 1.5 years } & \multicolumn{2}{|c|}{ At 2 years } & \\
\hline & EOP & LOP & EOP & LOP & EOP & LOP & EOP & LOP & \\
\hline Overall & $\begin{array}{l}2703 \\
(56.2)\end{array}$ & $\begin{array}{l}1009 \\
(56.6)\end{array}$ & $\begin{array}{l}2518 \\
(56.6)\end{array}$ & $\begin{array}{l}928 \\
(57.7)\end{array}$ & $\begin{array}{l}2282 \\
(56.7)\end{array}$ & $\begin{array}{l}818 \\
56.8)\end{array}$ & $\begin{array}{l}2145 \\
(55.7)\end{array}$ & $\begin{array}{l}773 \\
(57.7)\end{array}$ & $\begin{array}{l}1.14(1.05-1.25) \\
p=0.0019\end{array}$ \\
\hline Adalimumab & $\begin{array}{l}740 \\
(58.3)\end{array}$ & $\begin{array}{l}266 \\
(55.3)\end{array}$ & $\begin{array}{l}668 \\
(58.3)\end{array}$ & $\begin{array}{l}250 \\
(59.0)\end{array}$ & $\begin{array}{l}618 \\
(59.7)\end{array}$ & $\begin{array}{l}223 \\
(59.6)\end{array}$ & $\begin{array}{l}558 \\
(56.8)\end{array}$ & $\begin{array}{l}208 \\
(58.4)\end{array}$ & $\begin{array}{l}1.09(0.93-1.28) \\
p=0.2755\end{array}$ \\
\hline Etanercept & $\begin{array}{l}432 \\
(52.5)\end{array}$ & $\begin{array}{l}209 \\
(59.4)\end{array}$ & $\begin{array}{l}396 \\
(52.3)\end{array}$ & $\begin{array}{l}185 \\
(56.8)\end{array}$ & $\begin{array}{l}357 \\
(52.3)\end{array}$ & $\begin{array}{l}169 \\
(56.9)\end{array}$ & $\begin{array}{l}320 \\
(49.7)\end{array}$ & $\begin{array}{l}153 \\
(55.8)\end{array}$ & $\begin{array}{l}1.38(1.14-1.66) \\
p=0.0010\end{array}$ \\
\hline Infliximab & $\begin{array}{l}332 \\
(59.0)\end{array}$ & $\begin{array}{l}134 \\
(65.7)\end{array}$ & $\begin{array}{l}312 \\
(58.4)\end{array}$ & $\begin{array}{l}113 \\
(61.1)\end{array}$ & $\begin{array}{l}288 \\
(59.8)\end{array}$ & $\begin{array}{l}105 \\
(61.8)\end{array}$ & $\begin{array}{l}274 \\
(59.8)\end{array}$ & $\begin{array}{l}96 \\
(65.3)\end{array}$ & $\begin{array}{l}1.15(0.90-1.46) \\
p=0.2652\end{array}$ \\
\hline Ustekinumab & $\begin{array}{l}1139 \\
(57.4)\end{array}$ & $\begin{array}{l}309 \\
(55.5)\end{array}$ & $\begin{array}{l}1083 \\
(58.1)\end{array}$ & $\begin{array}{l}312 \\
(60.0)\end{array}$ & $\begin{array}{l}974 \\
(57.4)\end{array}$ & $\begin{array}{l}261 \\
(56.7)\end{array}$ & $\begin{array}{l}951 \\
(58.2)\end{array}$ & $\begin{array}{l}253 \\
(58.4)\end{array}$ & $\begin{array}{l}0.99(0.85-1.15) \\
p=0.8910\end{array}$ \\
\hline Methotrexate & $\begin{array}{l}59 \\
(35.1)\end{array}$ & $\begin{array}{l}90 \\
(48.1)\end{array}$ & $\begin{array}{l}58 \\
(38.9)\end{array}$ & $\begin{array}{l}67 \\
(44.1)\end{array}$ & $\begin{array}{l}45 \\
(36.0)\end{array}$ & $\begin{array}{l}59 \\
(42.8)\end{array}$ & $\begin{array}{l}41 \\
(31.8)\end{array}$ & $\begin{array}{l}62 \\
(48.4)\end{array}$ & $\begin{array}{l}1.62(1.16-2.26) \\
p=0.0049\end{array}$ \\
\hline
\end{tabular}

Data are presented as number of patients $(\%)$ or adjusted odds ratio (95\% confidence interval)

EOP early-onset psoriasis, LOP late-onset psoriasis, PGA 0/1 Physician's Global Assessment score of clear or minimal

Table 3 Patients achieving \%BSA $<3$ at post-baseline visits based on age of onset

\begin{tabular}{|c|c|c|c|c|c|c|c|c|c|}
\hline \multirow[t]{3}{*}{ Treatment } & \multicolumn{8}{|c|}{ Proportion of patients achieving $\% \mathrm{BSA}<3$} & \multirow{3}{*}{$\begin{array}{l}\text { Odds of achiev- } \\
\text { ing } \% \text { BSA }<3 \\
\text { for LOP vs. } \\
\text { EOP }\end{array}$} \\
\hline & \multicolumn{2}{|c|}{ At 6 months } & \multicolumn{2}{|c|}{ At 1 year } & \multicolumn{2}{|c|}{ At 1.5 years } & \multicolumn{2}{|c|}{ At 2 years } & \\
\hline & EOP & LOP & EOP & LOP & EOP & LOP & EOP & LOP & \\
\hline Overall & $\begin{array}{l}2857 \\
(59.4)\end{array}$ & $\begin{array}{l}1026 \\
(57.6)\end{array}$ & $\begin{array}{l}2765 \\
(62.2)\end{array}$ & $\begin{array}{l}1030 \\
(64.1)\end{array}$ & $\begin{array}{l}2591 \\
(64.4)\end{array}$ & $\begin{array}{l}920 \\
(63.9)\end{array}$ & $\begin{array}{l}2492 \\
(64.7)\end{array}$ & $\begin{array}{l}890 \\
(66.5)\end{array}$ & $\begin{array}{l}1.08(0.98-1.18) \\
p=0.1165\end{array}$ \\
\hline Adalimumab & $\begin{array}{l}772 \\
(60.8)\end{array}$ & $\begin{array}{l}276 \\
(57.4)\end{array}$ & $\begin{array}{l}734 \\
(64.1)\end{array}$ & $\begin{array}{l}266 \\
(62.7)\end{array}$ & $\begin{array}{l}681 \\
(65.8)\end{array}$ & $\begin{array}{l}238 \\
63.6)\end{array}$ & $\begin{array}{l}669 \\
(68.1)\end{array}$ & $\begin{array}{l}229 \\
(64.3)\end{array}$ & $\begin{array}{l}1.01(0.85-1.20) \\
p=0.9006\end{array}$ \\
\hline Etanercept & $\begin{array}{l}444 \\
(54.0)\end{array}$ & $\begin{array}{l}202 \\
(57.2)\end{array}$ & $\begin{array}{l}422 \\
(55.8)\end{array}$ & $\begin{array}{l}208 \\
(63.6)\end{array}$ & $\begin{array}{l}381 \\
(55.8)\end{array}$ & $\begin{array}{l}190 \\
(63.8)\end{array}$ & $\begin{array}{l}370 \\
(57.5)\end{array}$ & $\begin{array}{l}180 \\
(65.5)\end{array}$ & $\begin{array}{l}1.30(1.06-1.61) \\
p=0.0123\end{array}$ \\
\hline Infliximab & $\begin{array}{l}329 \\
(58.4)\end{array}$ & $\begin{array}{l}134 \\
(65.7)\end{array}$ & $\begin{array}{l}343 \\
(64.2)\end{array}$ & $\begin{array}{l}129 \\
(69.7)\end{array}$ & $\begin{array}{l}313 \\
(64.9)\end{array}$ & $\begin{array}{l}122 \\
(71.8)\end{array}$ & $\begin{array}{l}312 \\
(68.1)\end{array}$ & $\begin{array}{l}110 \\
(74.8)\end{array}$ & $\begin{array}{l}1.45(1.09-1.93) \\
p=0.0103\end{array}$ \\
\hline Ustekinumab & $\begin{array}{l}1241 \\
(62.5)\end{array}$ & $\begin{array}{l}325 \\
(58.4)\end{array}$ & $\begin{array}{l}1203 \\
(64.5)\end{array}$ & $\begin{array}{l}340 \\
(65.4)\end{array}$ & $\begin{array}{l}1159 \\
(68.3)\end{array}$ & $\begin{array}{l}300 \\
(65.2)\end{array}$ & $\begin{array}{l}1088 \\
(66.5)\end{array}$ & $\begin{array}{l}294 \\
(67.9)\end{array}$ & $\begin{array}{l}0.88(0.75-1.04) \\
p=0.1343\end{array}$ \\
\hline Methotrexate & $\begin{array}{l}71 \\
(42.3)\end{array}$ & $\begin{array}{l}89 \\
(47.6)\end{array}$ & $\begin{array}{l}63 \\
(42.3)\end{array}$ & $\begin{array}{l}87 \\
(57.2)\end{array}$ & $\begin{array}{l}57 \\
(45.6)\end{array}$ & $\begin{array}{l}70 \\
(50.7)\end{array}$ & $\begin{array}{l}53 \\
(41.1)\end{array}$ & $\begin{array}{l}77 \\
(60.2)\end{array}$ & $\begin{array}{l}1.27(0.87-1.84) \\
p=0.2120\end{array}$ \\
\hline
\end{tabular}

Data are presented as number of patients (\%) or adjusted odds ratio (95\% confidence interval)

$B S A$ body surface area, EOP early-onset psoriasis, $L O P$ late-onset psoriasis

CI 1.09-1.93]; $p=0.0103)$ and the likelihood of achieving $\% \mathrm{BSA}<1$ was $36 \%$ greater (AOR 1.36 [95\% CI 1.03-1.78]; $p=0.0290)$ than that of EOP patients. For patients treated with etanercept, those in the LOP group were $30 \%$ more likely to achieve \%BSA < 3 (AOR 1.30 [95\% CI 1.06-1.61]; $p=0.0123)$ and $34 \%$ more likely to achieve $\% \mathrm{BSA}<1(\mathrm{AOR}$
1.34 [95\% CI 1.09-1.64]; $p=0.0053$ ) than those in the EOP group. No difference was observed in the likelihood of achieving $\% \mathrm{BSA}<3$ or $\% \mathrm{BSA}<1$ for patients treated with ustekinumab, adalimumab, or methotrexate in the EOP and LOP groups (Tables 3 and 4). 
Table 4 Patients achieving \%BSA $<1$ at post-baseline visits based on age of onset

\begin{tabular}{|c|c|c|c|c|c|c|c|c|c|}
\hline \multirow[t]{3}{*}{ Treatment } & \multicolumn{8}{|c|}{ Proportion of patients achieving $\% \mathrm{BSA}<1$} & \multirow{3}{*}{$\begin{array}{l}\text { Odds of achiev- } \\
\text { ing \%BSA }<1 \\
\text { for LOP vs. } \\
\text { EOP }\end{array}$} \\
\hline & \multicolumn{2}{|c|}{ At 6 months } & \multicolumn{2}{|c|}{ At 1 year } & \multicolumn{2}{|c|}{ At 1.5 years } & \multicolumn{2}{|c|}{ At 2 years } & \\
\hline & EOP & LOP & EOP & LOP & EOP & LOP & EOP & LOP & \\
\hline Overall & $\begin{array}{l}2238 \\
(46.5)\end{array}$ & $\begin{array}{l}816 \\
(45.8)\end{array}$ & $\begin{array}{l}2209 \\
(49.7)\end{array}$ & $\begin{array}{l}801 \\
(49.8)\end{array}$ & $\begin{array}{l}1995 \\
(49.6)\end{array}$ & $\begin{array}{l}713 \\
(49.5)\end{array}$ & $\begin{array}{l}1992 \\
(51.8)\end{array}$ & $\begin{array}{l}723 \\
(54.0)\end{array}$ & $\begin{array}{l}1.09(1.00-1.19) \\
p=0.0582\end{array}$ \\
\hline Adalimumab & $\begin{array}{l}621 \\
(48.9)\end{array}$ & $\begin{array}{l}219 \\
(45.5)\end{array}$ & $\begin{array}{l}597 \\
(52.1)\end{array}$ & $\begin{array}{l}212 \\
(50.0)\end{array}$ & $\begin{array}{l}545 \\
(52.7)\end{array}$ & $\begin{array}{l}183 \\
(48.9)\end{array}$ & $\begin{array}{l}538 \\
(54.8)\end{array}$ & $\begin{array}{l}190 \\
(53.4)\end{array}$ & $\begin{array}{l}1.01(0.85-1.19) \\
p=0.9379\end{array}$ \\
\hline Etanercept & $\begin{array}{l}319 \\
(38.8)\end{array}$ & $\begin{array}{l}159 \\
(45.0)\end{array}$ & $\begin{array}{l}324 \\
(42.8)\end{array}$ & $\begin{array}{l}155 \\
(47.4)\end{array}$ & $\begin{array}{l}282 \\
(41.3)\end{array}$ & $\begin{array}{l}153 \\
(51.3)\end{array}$ & $\begin{array}{l}281 \\
(43.6)\end{array}$ & $\begin{array}{l}142 \\
(51.6)\end{array}$ & $\begin{array}{l}1.34(1.09-1.64) \\
p=0.0053\end{array}$ \\
\hline Infliximab & $\begin{array}{l}279 \\
(49.6)\end{array}$ & $\begin{array}{l}110 \\
(53.9)\end{array}$ & $\begin{array}{l}275 \\
(51.5)\end{array}$ & $\begin{array}{l}103 \\
(55.7)\end{array}$ & $\begin{array}{l}247 \\
(51.2)\end{array}$ & $\begin{array}{l}96 \\
(56.5)\end{array}$ & $\begin{array}{l}257 \\
(56.1)\end{array}$ & $\begin{array}{l}97 \\
(66.0)\end{array}$ & $\begin{array}{l}1.36(1.03-1.78) \\
p=0.0290\end{array}$ \\
\hline Ustekinumab & $\begin{array}{l}971 \\
(48.9)\end{array}$ & $\begin{array}{l}257 \\
(46.1)\end{array}$ & $\begin{array}{l}971 \\
(52.1)\end{array}$ & $\begin{array}{l}273 \\
(52.5)\end{array}$ & $\begin{array}{l}880 \\
(51.9)\end{array}$ & $\begin{array}{l}229 \\
(49.8)\end{array}$ & $\begin{array}{l}875 \\
(53.5)\end{array}$ & $\begin{array}{l}239 \\
(55.2)\end{array}$ & $\begin{array}{l}0.93(0.80-1.08) \\
p=0.3490\end{array}$ \\
\hline Methotrexate & $\begin{array}{l}48 \\
(28.6)\end{array}$ & $\begin{array}{l}71 \\
(38.0)\end{array}$ & $\begin{array}{l}42 \\
(28.2)\end{array}$ & $\begin{array}{l}58 \\
(38.2)\end{array}$ & $\begin{array}{l}41 \\
(32.8)\end{array}$ & $\begin{array}{l}52 \\
(37.7)\end{array}$ & $\begin{array}{l}41 \\
(31.8)\end{array}$ & $\begin{array}{l}55 \\
(43.0)\end{array}$ & $\begin{array}{l}1.35(0.92-1.97) \\
p=0.1241\end{array}$ \\
\hline
\end{tabular}

Data are presented as number of patients $(\%)$ or adjusted odds ratio (95\% confidence interval)

$B S A$ body surface area, EOP early-onset psoriasis, $L O P$ late-onset psoriasis

\section{Discussion}

Our findings are based on real-world data from 7511 patients with psoriasis who were treated with systemic therapies during enrollment in PSOLAR. Treatment outcomes were compared among EOP and LOP patients overall as well as among those treated with ustekinumab, adalimumab, etanercept, infliximab, and methotrexate at four time points ( 6 months, 1 year, 1.5 years, and 2 years). In this analysis, nearly three-quarters of patients (72.9\%) had EOP, which is consistent with previously reported prevalence data for EOP $[2,5]$. Furthermore, our results indicate that age of onset of psoriasis may affect how patients respond to systemic therapies based on measures of achieving PGA $0 / 1, \% \mathrm{BSA}<3$, or $\% \mathrm{BSA}<1$ responses.

The proportions of treatment responders in the EOP and LOP groups were consistent across measures assessed at each of four time points; however, the multivariate model detected significant differences in response between EOP and LOP groups for some treatments. Across all treatments combined, there was a $14 \%$ greater likelihood of LOP patients achieving PGA 0/1 response; however, no differences were observed in the proportion of patients achieving $\% \mathrm{BSA}<3$ or $\% \mathrm{BSA}<1$ responses. In contrast, sensitivity analyses by treatment showed that, compared with the EOP group, LOP patients responded better to etanercept, infliximab, and methotrexate. Patients in the LOP group who were treated with etanercept were 38 , 30 , and $34 \%$ more likely than those in the EOP group to have responded when response was measured using PGA $0 / 1, \% \mathrm{BSA}<3$, and $\% \mathrm{BSA}<1$, respectively. Similarly, infliximab-treated patients in the LOP group were $45 \%$ and $36 \%$ more likely than those in the EOP group to have achieved \%BSA $<3$ and \%BSA $<1$ responses, respectively; however, no difference was observed based on achieving PGA 0/1 response. Conversely, methotrexate-treated patients in the LOP group were $62 \%$ more likely to have achieved a PGA 0/1 response, but no difference was noted when assessed using \%BSA parameters. Therefore, our results for adalimumab, ustekinumab, and etanercept are consistent across measures and support the notion that adalimumab and ustekinumab are better choices than etanercept for treating EOP patients. The heterogeneity in responses between endpoints for infliximab and methotrexate complicate the interpretation, but the results generally suggest that these agents might be less useful for EOP patients than for those with LOP.

Response rates did not differ based on age of onset of psoriasis in patients treated with either ustekinumab or adalimumab. A possible explanation may be that patients who display different genetic susceptibility loci have variable treatment responses [8]. Studies evaluating response to biologics in psoriasis patients treated with ustekinumab and adalimumab have found variable responses among those carrying an HLA-Cw6 allele, which is associated with EOP, compared with those without this haplotype [9-13]. Other potential differences by age of onset, such as antidrug antibody development and treatment compliance patterns, may also have affected response rates across therapies.

As expected based on published literature, patients with EOP had a significantly greater disease duration (21.8 vs. 
8.3 years) and were more likely to have a positive family history of psoriasis (47.5 vs. $36.9 \%$ ) than patients with LOP. Patients in the EOP group in this analysis were also more likely than those in the LOP group to have been treated previously with oral systemic agents (34.8 vs. $29.3 \%$ ) and phototherapy (61.6 vs. $40.2 \%)$. Our findings are consistent with reports suggesting that, compared with patients with LOP, those with EOP may have an increased need for systemic therapies, given the greater disease severity and longer disease duration [14].

The findings of this analysis should be considered in the context of its limitations. The data used to draw these conclusions are derived from "real-world" clinical encounters that do not necessarily adhere to structured treatment protocols. Inherent to this limitation is the potential for treatment selection bias; however, the baseline disease severity for the two comparator groups (EOP vs. LOP) was not significantly different. Additionally, the statistical model may not account for all confounding variables.

\section{Conclusion}

Among the 7511 patients treated with biologics or methotrexate in PSOLAR, we found that age of onset may be associated with response to certain systemic treatments and may be useful for developing individualized treatments to maximize treatment response. Differences in responses between EOP and LOP patients was particularly evident for etanercept, infliximab, and methotrexate, suggesting those with LOP were more likely than those with EOP to experience a treatment response. However, the results suggested no difference in treatment outcomes in patients treated with ustekinumab or adalimumab.

Acknowledgements The authors thank Cynthia Arnold, BSc, CMPP (Janssen Scientific Affairs, LLC, Spring House, PA, USA) for her editorial assistance and writing support of this manuscript; Don Hoover, $\mathrm{PhD}$ (Data Solutions, LLC, and Rutgers University) for his statistical analysis support; and Joel Gelfand, MD, MSCE (Hospital of the University of Pennsylvania, Philadelphia) and the PSOLAR Scientific Advisory Committee, who provided critical review of the analytical plan.

\section{Compliance with Ethical Standards}

Funding This study was supported by Janssen Scientific Affairs, LLC, Horsham, PA, USA.

Conflicts of interest S. Singh has no conflicts of interest. R. E. Kalb has received grants and/or research funding from AbbVie, Amgen, Janssen, Merck \& Co, Inc., and Novartis Pharmaceuticals Corp.; served as a consultant for Dermira, Janssen-Ortho, Inc., Regeneron, and Sun Pharmaceutical Industries, Ltd.; and was a member of a Data Safety Monitoring Board for Eli Lilly and Company. E.M.G.J. de Jong has received research grants for the independent research fund of the
Dept. of Dermatology of the Radboud University Medical Center from AbbVie, Janssen, and Pfizer; and has acted as consultant and/or paid speaker for and/or participated in research sponsored by companies manufacturing drugs for the treatment of psoriasis, including AbbVie, Amgen, Celgene, Eli Lilly, Janssen, MSD, Novartis, and Pfizer (all funding goes to the independent research fund of "Radboud"). N. H. Shear has been a paid consultant for AbbVie, Actelion, Biogen, Celgene, Janssen, Leo, Lilly, Novartis, and Sanofi. M. Lebwohl is an employee of Mount Sinai, which receives research funds from AbbVie, Amgen, Boehringer Ingelheim, Celgene, Eli Lilly, Janssen/Johnson \& Johnson, Kadmon, Medimmune/Astra Zeneca, Novartis, Pfizer, and ViDac. B. Srivastava and L. Hopkins are employees of Janssen Scientific Affairs, LLC, and W. Langholff is an employee of Janssen Research \& Development, LLC. A.W. Armstrong has served as investigator, advisor, and/or consultant to AbbVie, Janssen, Novartis, Lilly, Regeneron, Sanofi, Leo, and Ortho Dermatologics.

Open Access This article is distributed under the terms of the Creative Commons Attribution-NonCommercial 4.0 International License (http://creativecommons.org/licenses/by-nc/4.0/), which permits any noncommercial use, distribution, and reproduction in any medium, provided you give appropriate credit to the original author(s) and the source, provide a link to the Creative Commons license, and indicate if changes were made.

\section{References}

1. Parisi R, Symmons DPM, Griffiths CEM, Asheroft DM. Global epidemiology of psoriasis: a systematic review of incidence and prevalence. J Invest Dermatol. 2013;133:377-85.

2. Henseler T, Christophers E. Psoriasis of early and late onset: characterization of two types of psoriasis vulgaris. J Am Acad Dermatol. 1985;13:450-6.

3. Zhao YE, Ma JX, Hu L, Xiao SX, Zhao YL. Meta-analysis of the association between psoriasis and human leukocyte antigen- $\mathrm{B}$. $\mathrm{Br}$ J Dermatol. 2013;169:417-27.

4. Ferrandiz C, Pujol RM, Garcia-Patos V, Bordas X, Smandia JA. Psoriasis of early and late onset: a clinical and epidemiologic study from Spain. J Am Acad Dermatol. 2002;46:867-73.

5. Griffiths CEM, Christophers E, Szumski A, Jones H, Mallbris L. Impact of early vs. late disease onset on treatment response to etanercept in patients with psoriasis. Br J Dermatol. 2015;173:1271-3.

6. Papp KA, Strober B, Augustin M, Calabro S, Londhe A, Chevrier M. PSOLAR: design, utility, and preliminary results of a prospective, international, disease-based registry of patients with psoriasis who are receiving, or are candidates for, conventional systemic treatments or biologic agents. J Drugs Dermatol. 2012;11:1210-7.

7. Kimball AB, Leonardi C, Stahle M, Gulliver W, Chevrier M, Fakharzadeh S, et al. Demography, baseline disease characteristics and treatment history of patients with psoriasis enrolled in a multicentre, prospective, disease-based registry (PSOLAR). Br J Dermatol. 2014;171:137-47.

8. van Vugt LJ, van den Reek JMPA, Coenen MJH, de Jong EMGJ. A systematic review of pharmacogenetic studies on the response to biologics in patients with psoriasis. Br J Dermatol. 2018;178:e80.

9. Gulliver WP, Young H, Gulliver S, Randell S. HLA-Cw6 status predicts efficacy of biologic treatments in psoriasis patients. Glob Dermatol. 2015;2:228-31.

10. Gallo E, Cabaleiro T, Roman M, Solano-Lopez G, Abad-Santos F, Garcia-Diez A, et al. The relationship between tumour necrosis factor (TNF)- $\alpha$ promoter and IL12B/IL-23R genes polymorphisms 
and the efficacy of anti-TNF- $\alpha$ therapy in psoriasis: a case-control study. Br J Dermatol. 2013;169:819-29.

11. Chiu HY, Wang TS, Chan CC, Cheng YP, Lin SJ, Tsai TF. Human leucocyte antigen-Cw6 as a predictor for clinical response to ustekinumab, an interleukin-12/23 blocker, in Chinese patients with psoriasis: a retrospective analysis. Br J Dermatol. 2014;171:1181-8.

12. Li K, Huang CC, Randazzo B, Li S, Szapary P, Curran M, et al. HLA-C*06:02 allele and response to IL-12/23 inhibition: results from the ustekinumab phase 3 psoriasis program. J Investig Dermatol. 2016;136:2364-71.
13. Talamonti M, Botti E, Galluzzo M, Teoli M, Spallone G, Bavetta $\mathrm{M}$, et al. Pharmacogenetics of psoriasis: HLA-Cw6 but not LCE3B/3C deletion nor TNFAIP3 polymorphism predisposes to clinical response to interleukin 12/23 blocker ustekinumab. Br J Dermatol. 2013;169:458-63.

14. Di Lernia V, Ficarelli E. Current therapeutic approaches of psoriasis are affected by age at disease onset. J Dermatol Treat. 2014;25:15-7. 